\title{
Sensitivity and frequencies of dystrophin gene mutations in Thai DMD/BMD patients as detected by multiplex PCR
}

\author{
Thanyachai Sura ${ }^{1}$, Jakris Eu-ahsunthornwattana ${ }^{1, *}$, Sarinee Pingsuthiwong and Manisa Busabaratana \\ Division of Medical Genetics \& Molecular Medicine and Academic Center for Medical Genetics, Department of \\ Medicine, Faculty of Medicine Ramathibodi Hospital, Mahidol University, Bangkok, Thailand
}

\begin{abstract}
Background: Duchenne muscular dystrophy (DMD), a lethal X-linked disease affecting 1 in 3500 male births, and its more benign variant, Becker muscular dystrophy (BMD), are caused by mutations in the dystrophin gene. Because of its large size, analysing the whole gene is impractical. Methods have been developed to detect the commonest mutations i.e. the deletions of the exons. Although these tests are highly specific, their sensitivity is inherently limited by the prevalence of deletions, which differs among different populations.

Methods: We reviewed our database for the detection of Dystrophin gene mutation by means of 31-exon multiplex PCR in Thai males, diagnosed clinically and biochemically with DMD or BMD from July 1994 to November 2006. One index patient was chosen from each family for statistical analysis. The overall sensitivity of the test, the number of fragment deleted, and the deletion frequency of each fragment were calculated, along with their $95 \%$ confidence intervals (C.I.).

Results: We found deletions in 99 out of the 202 index patients (49\%; Bayesian 95\% C.I. $=42 \%-56 \%$ ). $51 \%$ of these had deletion in only one of the 31 exons tested, while the patient with the most extensive deletions had 14 exons deleted. The mean number of deleted exons were $2.84\left(\mathrm{BC}_{\mathrm{a}}\right.$ bootstrap 95\% C.I. $\left.=2.37-3.48\right)$, or $5.02(3.81-6.85)$ if all the untested exons adjacent to the confirmed deleted exons were assumed to be deleted. The region spanning exons 44-52 was the most frequently deleted. These were similar to those reported in the Japanese.

Conclusion: The multiplex PCR detected deletions only in about half of the Thai patients. The diseases therefore should not be excluded solely on the negative result if DMD/BMD is strongly suspected.
\end{abstract}

Keywords: Duchenne muscular dystrophy, dystrophin, gene deletion, multiplex PCR, sensitivity, Thai people

\section{Introduction}

Duchenne muscular dystrophy (DMD; OMIM 310200), the most common lethal X-linked disorder in men, affects approximately 1 in 3500 male births. Both the DMD and its much milder variant, Becker muscular dystrophy (BMD; OMIM 300376), are caused by mu-

\footnotetext{
${ }^{1}$ Co-first authors.

* Corresponding author: Dr. Jakris Eu-ahsunthornwattana, Division of Medical Genetics \& Molecular Medicine, Department of Medicine, Ramathibodi Hospital, Rama VI Rd., Phaya Thai, Bangkok 10300, Thailand. Office Tel./Fax: +66 2201 1374; Home Tel.: +66 87912 7847; E-mail: jakris_e@iname.com.
}

tations in the dystrophin (DMD) gene. With 79 exons spanning about 2.6 million base pairs, the gene is the largest yet identified in the human genome [17,27].

Based on the Southern hybridization with cDNA probes in the caucasians, approximately $50 \%-70 \%$ of the mutations causing DMD have been found to be gross deletions, while about $5 \%$ were believed to be duplications, and the remaining were point mutations [4, 11,16,21]. These DMD-producing mutations are believed to cause downstream frameshift error. BMD, on the other hand, are believed to be caused by mutations that still maintain the reading frame, result only in reduction of the amount or function of the product, and hence milder phenotype [4]. 
Because of the size of the gene and the predominantly deletional nature of the mutations, direct searching for mutations in the dystrophin gene is very expensive. Several techniques have therefore been developed to aid in detecting common mutations more economically. Multiplex polymerase chain reaction (PCR) has the benefit of being minimally invasive, efficient and very specific for the detection of large gene deletions, and has superseded the more laborious Southern blot method since the early '90s [6]. While being recently replaced by more modern techniques such as the Multiple Ligation-dependent Probe Amplification (MLPA) in many laboratories, the Multiplex-PCR still benefits from its requirement of relatively simpler equipments. Using the 19-exons primers combination as described by Chamberlain et al [8,9] and themselves, Beggs et al reported detection rate of as high as $98 \%$ of the DMD/BMD deletions detected by the Southern blot analysis [5]. Nevertheless, since the test detects only the common deletions and will miss the rare deletions, point mutations or duplications [30], its overall sensitivity in any population will only be as high as the prevalence of large deletions among its DMD/BMD patients. This has been reported to differ among various populations [29]. In Asian populations, using the same 19-exons set, the detection rate could well be substantially lower: $40 \%$ in Singaporeans or $32 \%$ in Vietnamese. This could reflect the higher proportion of deletions outside of the 'hotspots' [22] or the different nature of the predominant mutations e.g. duplications or point mutations.

Little is known about the prevalence of deletions among the Thai DMD/BMD patients. Since this information is population specific and is necessary in clinical counselling, particularly in the prenatal diagnosis scenario; we conducted this study to assess the sensitivity of the multiplex PCR for DMD/BMD in Thai population, and to ascertain the pattern of deletion and compare this with those from other Southeast Asian population.

\section{Methods}

\subsection{Subjects}

Our medical genetic division has offered molecular diagnostic service for Duchenne muscular dystrophy since 1994. We reviewed our laboratory records to identify an index patient from each family with a clinical diagnosis of either DMD or BMD, based on the clinical criteria [29] and serum creatine kinase enzyme level [19]. We excluded subjects with any of the following characteristics: (a) subjects whose clinical features or serum creatine kinase levels were not consistent with DMD or BMD according to the above criteria; (b) subjects whose most likely diagnosis was not DMD/BMD but had their samples sent for "exclusion" purpose; or (c) subjects whose affected family member had already been counted as index case in this study. We identified 202 index subjects using these criteria.

\subsection{The multiplex $P C R$}

The subjects' genomic DNA was extracted from their EDTA-anticoagulated peripheral blood samples upon receipt using standard phenol-chloroform method, and later used in the multiplex polymerase chain reaction (multiplex PCR). We modified the multiplex PCRs described by Beggs [6] and Curtis and Haggerty [10] to detect deletions in 31 exons and promoter regions (brain-specific promoter $(p \mathrm{~b})$, muscle-specific promoter together with exon $1(\mathrm{pm})$, exons 2 (part 1), 3-6, 7 (part 1), 8, 12, 13, 16, 17, 19, 20, 32, 34, 41-53, 60) of the dystrophin gene.

To facilitate the result interpretation, Beggs' diagnostic sets A, B and C were divided to A1, A2, B1, B2, $\mathrm{C} 1$, and $\mathrm{C} 2$, containing the primers for the following exons: (A1): 8, 17, 44, 46, 48; (A2): 4, 12, 19, 45, 51; (B1): $p \mathrm{~m}, 13,43,47,52$; (B2): 3, 6, 50, 60; (C1): 16, 32, 34, 49; and (C2): pb, 41, 42. We combined these with a selection of primers as described by Curtis and Haggerty as follows: the primers pair for exon 7 (part 1) was added to the diagnostic set $\mathrm{C} 2$; the primers for four more exons were divided into two more diagnostic sets, each contained the following exons: (D1): 5, 53; (D2): 2 (part 1), 20.

Eight multiplex PCRs were performed for each subject, using the primer sets described. The PCR conditions were: sets A1-C2, initial denaturation at $95^{\circ} \mathrm{C}$ for 2 minutes followed by 35 cycles of denaturation at $95^{\circ} \mathrm{C}$ for 1 minutes, annealing at $65^{\circ} \mathrm{C}$ for 1 minutes, and extension at $72^{\circ} \mathrm{C}$ for 4 minutes, with a final extension for a further 6 minutes at $72^{\circ} \mathrm{C}$; sets D1 and $\mathrm{D} 2$, initial denaturation at $94^{\circ} \mathrm{C}$ for 5 minutes followed by 35 cycles of denaturation at $94^{\circ} \mathrm{C}$ for 1 minutes, annealing at $53^{\circ} \mathrm{C}$ for 1 minutes, and extension at $72^{\circ} \mathrm{C}$ for 1 minutes, with a final extension for a further 7 minutes at $72^{\circ} \mathrm{C}$. The PCR products were analysed using standard agarose gel electrophoresis, visualised under an ultraviolet transilluminator. 


\subsection{Data entry and analysis}

The test results along with the individual and family identification data was entered into a spreadsheet application. Validation and cleaning was done prior to importation into R 2.4.1 [28] for statistical analysis. After initial data integrity check, we calculated the proportion of the positive results in each subgroups (DMD/BMD) as well as in all patients with its equaltailed Bayesian 95\% confidence interval (C.I.), using the uninformative Jeffreys prior [7]. For the percentage of the patients who had each particular fragment deleted, the confidence intervals were calculated using the Agresti-Coull method [1]. The bias-corrected and accelerated $\left(\mathrm{BC}_{\mathrm{a}}\right)$ bootstrap method [12] was used for the estimation of the average number of exons deleted and its confidence interval.

\section{Results}

Of the 202 index patients identified, 199 had been diagnosed with DMD and the remaining 3 patients with BMD. We detected deletions in 99 of the 202 index patients (49\%; Jeffreys prior 95\% C.I. $=42 \%-56 \%$ ) as shown in Table 1 (see also the bottom plot of Fig. 1). For comparison, we would have detected deletions in 91 patients $(45 \%$; $95 \%$ C.I. $=38 \%-52 \%)$ had we used the 19-exons PCR as in the previously mentioned studies $[5,8,9,22]$. Fifty of the 99 patients with detectable deletions had deletion in only one of the 31 tested exons. Among those whose deletions could be detected, the mean size of dystrophin deletion ranged from 2.84 exons $\left(\mathrm{BC}_{\mathrm{a}}\right.$ bootstrap 95\% C.I. $\left.=2.37-3.48\right)$, assuming no further deletion in the untested area, to 5.02 exons (3.81-6.85) assuming that all the untested exons adjacent to any deleted exon were also deleted. The patient with longest deletion lost 14 of the tested exons. This could be as extensive as 38 exons if we were to assume deletion in the untested exons adjacent to the confirmed deletion.

The proportion of patients who have deletion involving each exon is shown in Fig. 2. The region spanning exons 44-52 was where the deletions occurred most frequently, followed by exons 8-19 and 3-6. The extent of deletions in each patient is shown in Fig. 3. The deletions in the distal hot spot (exons 44-52) accounted for approximately $59 \%$ of the deletions, while the deletions in the proximal hot spot (exons 3-19) accounted for about $30 \%$.

Three of the DMD patients whose tests were negative had the Southern blot done, and were found to have a mutation in exon 42,49 , and 51 , respectively.

\section{Discussion}

Partial deletions were detected in approximately $49 \%$ of our cases $(95 \%$ C.I. $42 \%-56 \%)$, or $45 \%(38 \%$ $52 \%$ ) if the original Chamberlain and Beggs 19-exon primer subset had been used. Compared with other populations in this region, the detection rate in our study is comparable to the reported rate in the Japanese [22]. It was slightly higher than those in Singaporean and Vietnamese [22] and lower than that in the Chinese [31] or Indians [2,3,24,29]. Compared with the previous studies using similar methods in other unrelated populations, our detection rate was similar to that reported in the Czechs [18], slightly lower than those reported in other Caucasians [6,26] and the Egyptians [13], and much lower than that in the Brazilians [15] (Fig. 1).

The discrepancy could be due to many factors. Firstly, the numbers of the tested exons varied among the studies. There could also be subtle variation in the methods used among the laboratories, which may affect the diagnostic yields. Of note, one study [15] actually used the non-multiplex version of the test, and could possibly have less interference. All studies used clinical and serum creatine kinase criteria, but some also required positive muscle biopsy. Since our study did not require muscle biopsy for inclusion, the sensitivity could be negatively biased as will be discussed below. Finally, but perhaps more importantly, it may reflect the different prevalence for large deletions or different deletional pattern in different populations as has previously been demonstrated [29].

In our own population, two small studies using subsets of these primers previously reported the detection rate of $54 \%$ ( 7 out of 13 unrelated cases, covering the 10 exons described by Beggs et al. [5]; 95\% Bayesian interval 29\%-78\%) [20] and 56\% (5 out of 9 cases, using the 9-exon set described by Chamberlain et al. [9]; $95 \%$ C.I. 26\%-83\%) [25]. Since one of these required positive muscle biopsy, and most of the subjects in the other had positive muscle biopsy, we believe the sensitivity of the 31-exon multiplex-PCR in Thai patients in whom the DMD or BMD is suspected is approximately $50 \%$.

Our estimation of the sensitivity is likely to be conservative because muscle biopsy was not done or not available in some of the included patients. It is therefore possible that some of these patients may in fact not be affected from DMD/BMD, thus negatively biased the estimated sensitivity. Naturally, our estimated sensitivity will be less than those from the studies where muscle biopsy was mandatory. However, as there is 
Table 1

Proportion of patients with detectable deletions in the Dystrophin gene

\begin{tabular}{lrcl}
\hline Diagnosis & Total & Number detected & Percentage \\
\hline DMD & 199 & 98 & $49 \%(49 \% ; 42 \%-56 \%)$ \\
BMD & 3 & 1 & $33 \%(38 \% ; 1 \%-77 \%)$ \\
All & 202 & 99 & $49 \%(49 \% ; 42 \%-56 \%)$ \\
\hline
\end{tabular}

Numbers in brackets are the Jeffreys prior Bayesian estimates of the percentages and the $95 \%$ confidence intervals.

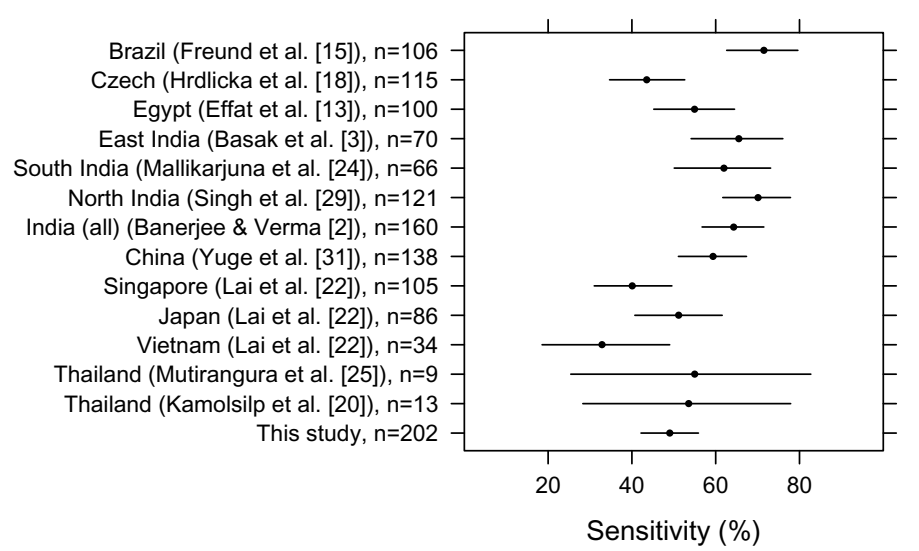

Fig. 1. Sensitivity of dystrophin multiplex PCR in various populations. The dots represent the Bayesian estimate of sensitivity derived from each study, with the horizontal lines representing their $95 \%$ Bayesian intervals.

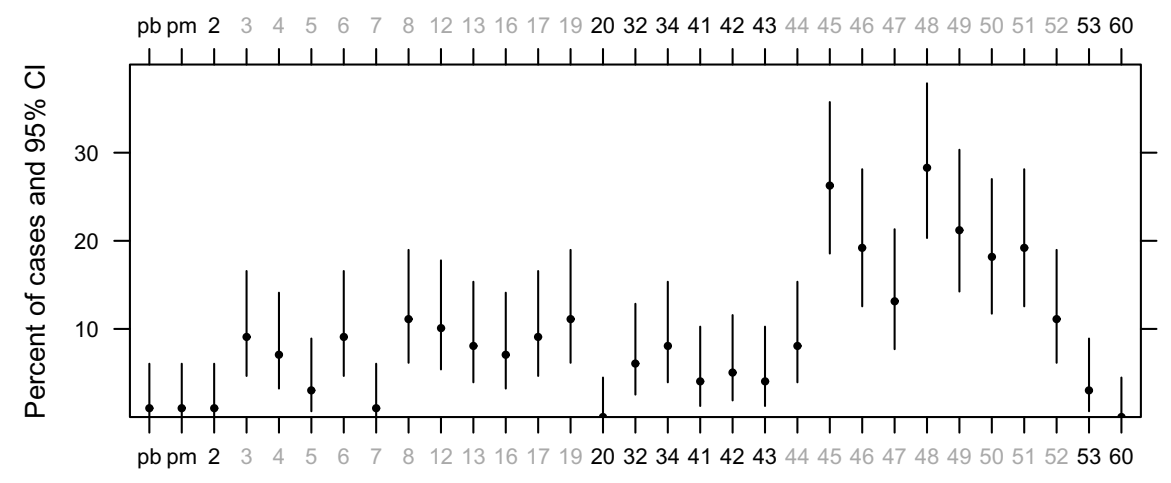

Fragment deleted

Fig. 2. Percentage of patients with detectable deletions who have each particular fragment deleted. Each data point shows the percentage of patients who have a particular fragment of Dystrophin gene deleted. Exons marked in grey were those within the hotspots. The vertical lines represent the $95 \%$ confidence intervals of the estimated percentage as calculated using the Agresti-Coull method, with the lower boundaries clipped at zero.

a preference to avoid the muscle biopsy in favour of the less invasive molecular genetic methods [15], we believe our estimated detection rate to be similar to that seen in current practice.

In an ideal situation, the estimated sensivity will be equal to the prevalence of deletion in the population. However, our inference of the prevalence of deletions among the DMD/BMD patients could be negatively bi- ased by the fact that we have tested for just 31 out of the total of 79 exons. Nevertheless, the effect is likely to be small since previous studies that directly compared the multiplex PCR to other methods in the same subjects have shown that the multiplex PCR covering the two hotspots could detect more than $95 \%$ of the deletions detected by the whole gene cDNA probes $[5,29,31]$ or MLPA [23]. Additionally, the detection rates of the 


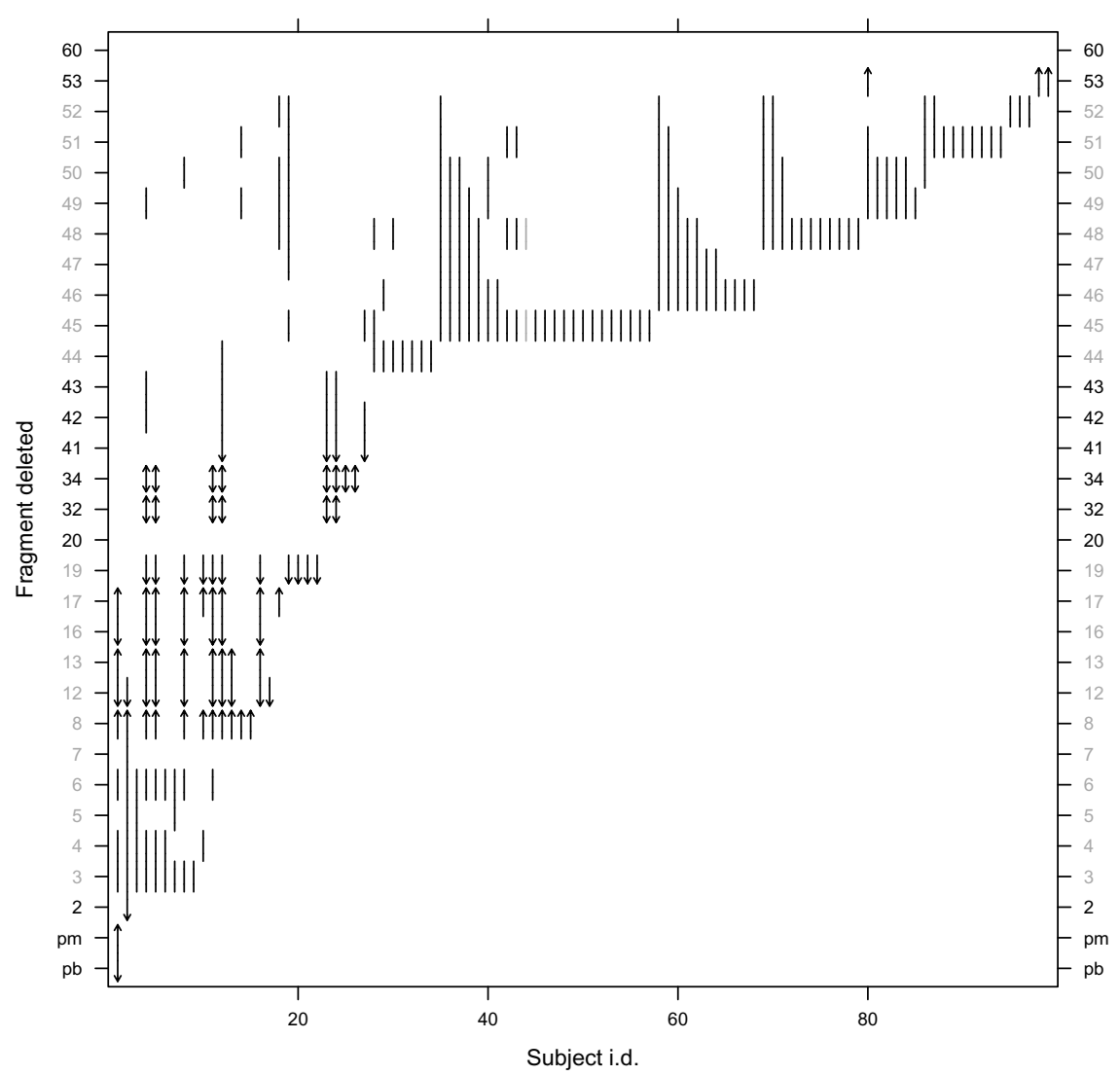

Fig. 3. Deletional pattern in each subject. The vertical bar(s) represent the deleted fragment(s) in each patient. All were diagnosed with DMD except patient number 44 (in grey) who was diagnosed with BMD. The exon numbers marked in grey were those within the hotspots. An arrow indicates that the extent of deletion could be anywhere from that exon to the next tested exon. For example, subject no. 16 had confirmed deletions in exons $12,13,16,17$, and 19 ; exons 14,15 , and 18 were not tested for, although it is likely that these are also involved which will make this a single contiguous deletion spanning from at least exon 12 to 19 . The beginning of the deletion could not be exactly determined since exons 9-11 were not tested for; it could therefore be anywhere from exon 9 to 12 . On the contrary, the last exon deleted was definitely exon 19 since exon 20 was presented in the multiplex-PCR reaction.

deletion from studies using multiplex PCR have also been shown to be similar to those from studies using cDNA probes in the same populations [2]. Assuming similar pattern of deletions to other populations, we believe the prevalence of large deletions among Thai patients diagnosed with DMD/BMD on the clinical and biochemical grounds to be in the range of $50 \%-60 \%$.

It is apparent from Fig. 2 that deletions among different exons occurred with unequal frequencies. This inequality has previously been reported. Deletions in the dystrophin gene occur mostly in two high-frequency deletion regions: the distal (or central) hot spot, located centrally about $1200 \mathrm{~kb}$ from exon 1 and encompassing exons 44-53, is responsible for about two-third of the deletions; and the proximal hot spot which extends from exon 1 to 19 is responsible for a third [4,5,14,21, 22]. This was thought to be due to the varying size of the introns, and the presence of local recombination hot spots [16]. The pattern of deletions found in our study is similar to those previously reported, particularly the Japanese population [22].

Another method, the Multiplex Ligation-dependent Probe Amplification (MLPA), have been developed over the past few years, and is very likely replace the multiplex PCR as a method of choice for the detection of dystrophin mutations due to its superior sensitivity. However, despite its superiority, the test will detect just the deletions and duplications but not the point mutations unless occur at the hybridisation site. Given the relatively low prevalence of large deletion in this region as demonstrated in the previous [22] and this study, we suspect that the sensitivity of the MLPA for the detection of dystrophin mutation in the Southeast Asian population, although will definitely be better, may not 
be very high: probably in the range of $55 \%-65 \%$ based on the above estimation of the deletional frequency and the estimated duplication frequency of 5\% from other populations. In the mean time, users of the multiplex PCR results should keep in mind that the test will give a false-negative rate of about $50 \%$. As with any other tests, the result of multiplex PCR should always be used in conjunction with the prior probability, based on good clinical history and examinations, and confirmed by alternative test when necessary.

\section{Acknowledgment}

This project was partially supported by the Medical Genetics Research Fund, Ramathibodi Foundation, Faculty of Medicine Ramathibodi Hospital, Mahidol University, Bangkok, Thailand. We would like to thank Associate Professor Supoj Tulyadachanon who had assisted with the initial setup of this test; Drs. Atchara Tunteeratum, Objoon Trachoo, Donniphat Dejsuphong, Supischa Theerasasawat, and Ms Kanoknan Srichan who had helped in collecting the patients; and Artit Sripirom and Supak Youngcharoen who had helped with some of these tests.

\section{References}

[1] A. Agresti and B.A. Coull, Approximate is better than "exact" for interval estimation of binomial proportions, Amer Statist 52 (1998), 119-126.

[2] M. Banerjee and I.C. Verma, Are there ethnic differences in deletions in the dystrophin gene? Am J Med Genet 68 (1997), 152-157.

[3] J. Basak, U.B. Dasgupta, T.K. Banerjee, A.K. Senapati, S.K. Das and S.C. Mukherjee, Analysis of dystrophin gene deletions by multiplex PCR in eastern India, Neurol India $\mathbf{5 4}$ (2006), 310-311.

[4] L.L. Baumbach, J.S. Chamberlain, P.A. Ward, N.J. Farwell and C.T. Caskey, Molecular and clinical correlations of deletions leading to Duchenne and Becker muscular dystrophies, Neurol 39 (1989), 465-474.

[5] A.H. Beggs, M. Koenig, F.M. Boyce and L.M. Kunkel, Detection of $98 \%$ of DMD/BMD gene deletions by polymerase chain reaction, Hum Genet 86 (1990), 45-48.

[6] A.H. Beggs, Multiplex PCR for identifying dystrophin gene deletions, in: Current Protocol in Human Genetics, (Vol. 2), N.C. Dracopoli, J.L. Haines, B.R. Korf, D.T. Moir, C.C. Morton, C.E. Seidman, J.G. Seidman and D.R. Smith, eds, John Wiley \& Sons, New York, 1994, pp. 9.3.1-9.3.17.

[7] L.D. Brown, T. Cai and A. DasGupta, Interval estimation for a binomial proportion, Statistical Sci 16 (2001), 101-117.

[8] J.S. Chamberlain, R.A. Gibbs, J.E. Ranier, P.N. Nguyen and C.T. Caskey, Deletion screening of the Duchenne muscular dystrophy locus via multiplex DNA amplification, Nucleic Acids Res 16 (1988), 11141-11156.
[9] J.S. Chamberlain, R.A. Gibbs, J.E. Ranier and C.T. Caskey. Multiplex PCR for the diagnosis of Duchenne muscular dystrophy, in: PCR Protocols: A Guide to Methods and Applications, M.A. Innis, D.H. Gelfand, J.J. Sninsky and T.J. White, eds, Academic Press, New York, 1990, pp. 272-281.

[10] A. Curtis and D. Haggerty, Deletion and duplication analysis in males affected with Duchenne or Becker muscular dystrophy, in: Muscular Dystrophy: Methods and Protocols, K.M.D. Bushby and L.V.B. Anderson, eds, Humana Press, Totowa, NJ, 2001, pp. 53-84.

[11] J.T. den Dunnen, P.M. Grootscholten, E. Bakker, L.A. Blonden, H.B. Ginjaar, M.C. Wapenaar, H.M. van Paassen, C. van Broeckhoven, P.L. Pearson and G.J. van Ommen, Topography of the Duchenne muscular dystrophy (DMD) gene: FIGE and cDNA analysis of 194 cases reveals 115 deletions and 13 duplications, Am J Hum Genet 45 (1989), 835-847.

[12] T.J. DiCiccio and B. Efron, Bootstrap confidence intervals, Statistical Science 11 (1996), 189-212.

[13] L.K. Effat, A.A. El-Harouni, K.S. Amr, T.I. El-Minisi, N. Abdel Meguid and M. El-Awady, Screening of dystrophin gene deletions in Egyptian patients with DMD/BMD muscular dystrophies, Dis Markers 16 (2000), 125-129.

[14] S.M. Forrest, G.S. Cross, T. Flint, A. Speer, K.J.H. Robson and K.E. Davies, Further studies of gene deletions that cause Duchenne and Becker muscular dystrophies, Genomics 2 (1988), 109-114.

[15] A.A. Freund, R.H. Scola, R.C. Arndt, P.J. Lorenzoni, C.K. Kay and L.C. Werneck, Duchenne and Becker muscular dystrophy: a molecular and immunohistochemical approach, Arq Neuropsiquiatr 65 (2007), 73-76.

[16] E.F. Gillard, J.S. Chamberlain, E.G. Murphy, C.L. Duff, B. Smith, A.H.M. Burghes, M.W. Thompson, J. Sutherland, I. Oss, S.E. Bodrug, H.J. Klamut, P.N. Ray and R.G. Worton, Molecular and phenotypic analysis of patients with deletions within the deletion-rich region of the Duchenne muscular dystrophy (DMD) gene, Am J Hum Genet 45 (1989), 507-520.

[17] E.P. Hoffman and D. Dressman, Molecular pathophysiology and targeted therapeutics for muscular dystrophy, Trends Pharmacol Sci 22 (2001), 465-470.

[18] I. Hrdlicka, J. Zadina, R. Krejcí, A. Srbová and M. Kucerová, Patterns of deletions and the distribution of breakpoints in the dystrophin gene in Czech patients with Duchenne and Becker muscular dystrophy (statistical comparison with results from several other countries), Folia Biol (Praha) 47 (2001), 81-87.

[19] F.G. Jennekens, L.P. ten Kate, M. de Visser and A.R. Wintzen, Diagnostic criteria for Duchenne and Becker muscular dystrophy and myotonic dystrophy, Neuromuscul Disord 1 (1991), 389-391.

[20] M. Kamolsilp, R. Paditaporn, A. Noonai and P. Wasant, Detection of DMD gene deletions in Thai children patients, Southeast Asian J Trop Med Public Health 26(Suppl 1) (1995), 172-174.

[21] M. Koenig, E.P. Hoffman, C.J. Bertelson, A.P. Monaco, C. Feener and L.M. Kunkel, Complete cloning of the Duchenne muscular dystrophy (DMD) cDNA and preliminary genomic organization of the DMD gene in normal and affected individuals, Cell 50 (1987), 509-517.

[22] P.S. Lai, Y. Takeshima, K. Adachi, K. Van Tran, H.T. Nguyen, P.S. Low and M. Matsuo, Comparative study on deletions of the dystrophin gene in three Asian populations, Hum Genet 47 (2002), 552-555.

[23] K.K.S. Lai, I.F.M. Lo, T.M.F. Tong, L.Y.L. Cheng and S.T.S. Lam, Detecting exon deletions and duplications of the DMD 
gene using Multiplex Ligation-dependent Probe Amplification (MLPA), Clin Biochem 39 (2005), 367-372.

[24] G.N. Mallikarjuna Rao, T. Hussain, N. Geetha Devi, S. Jain, G.R. Chandak and M.P. Ananda Raj, Dystrophin gene deletions in South Indian Duchenne muscular dystrophy patients, Indian J Med Sci 57 (2003), 1-6.

[25] A. Mutirangura, S. Jongpiputvanich, T. Norapucsunton, A. Theamboonlers, S. Srivuthana, C. Promchainant, S. Tumwasorn and T. Sueblinvong, Multiplex PCR to detect the dystrophin gene deletion in Thai patients, J Med Assoc Thai $\mathbf{7 8}$ (1995), 460-465.

[26] S. Niemann-Seyde, R. Slomski, F. Rininsland, U. Ellermeyer, J. Kwiatkowska and J. Reiss, Molecular genetic analysis of 67 patients with Duchenne/Becker muscular dystrophy, Hum Genet 90 (1992), 65-70.

[27] K.J. Nowak and K.E. Davies, Duchenne muscular dystrophy and dystrophin: pathogenesis and opportunities for treatment, EMBO Reports 5 (2004), 872-876.
[28] R Development Core Team, $R$ : A language and environment for statistical computing, R Foundation for Statistical Computing, Vienna, Austria, 2006. [Available online at URL: http://www.R-project.org].

[29] V. Singh, S. Sinha, S, Mishra, L.S. Chaturvedi, S. Pradhan, R.D. Mittal and B. Mittal, Proportion and pattern of dystrophin gene deletions in North Indian Duchenne and Becker muscular dystrophy patients, Hum Genet 99 (1997), 206-208.

[30] S.K.H. Tay, H.H. Khng, P.S. Low and P.S. Lai, Diagnostic Strategy for the detection of dystrophin gene mutations in Asian patients and carriers using immortalized cell lines, $J$ Child Neurol 21 (2006), 150-155.

[31] L. Yuge, L. Hui and X. Bingdi, Detection of gene deletions in Chinese patients with Duchenne/Becker muscular dystrophy using cDNA probes and the polymerase chain reaction method, Life Sci 65 (1999), 863-869. 


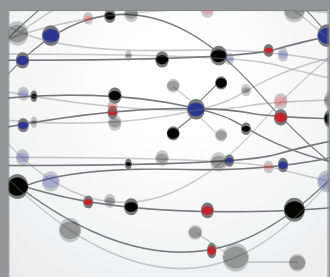

The Scientific World Journal
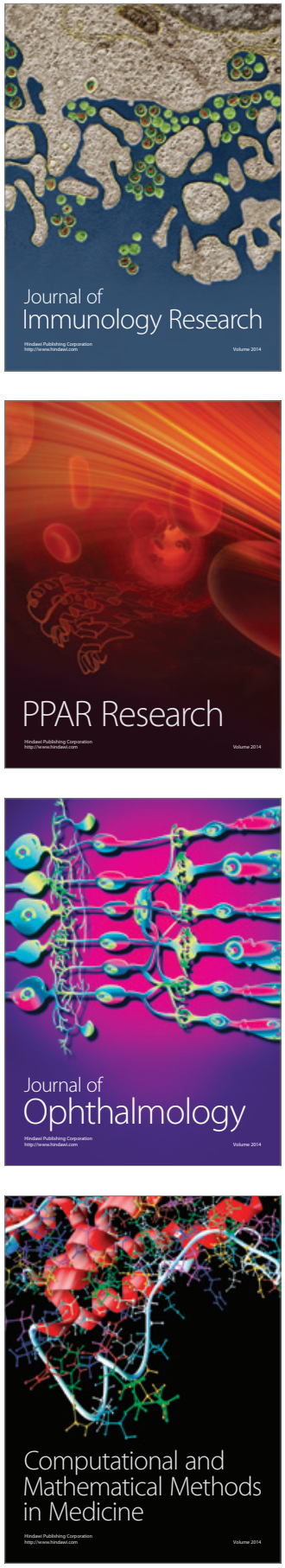

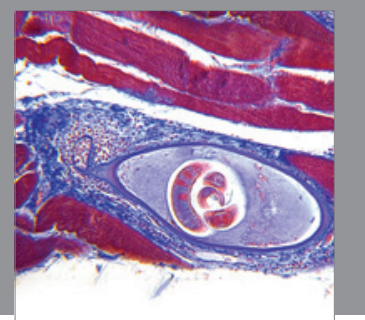

Gastroenterology

Research and Practice
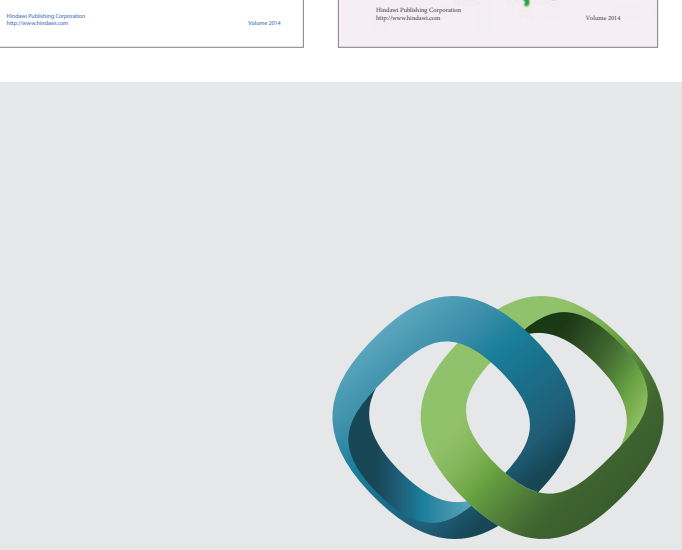

\section{Hindawi}

Submit your manuscripts at

http://www.hindawi.com
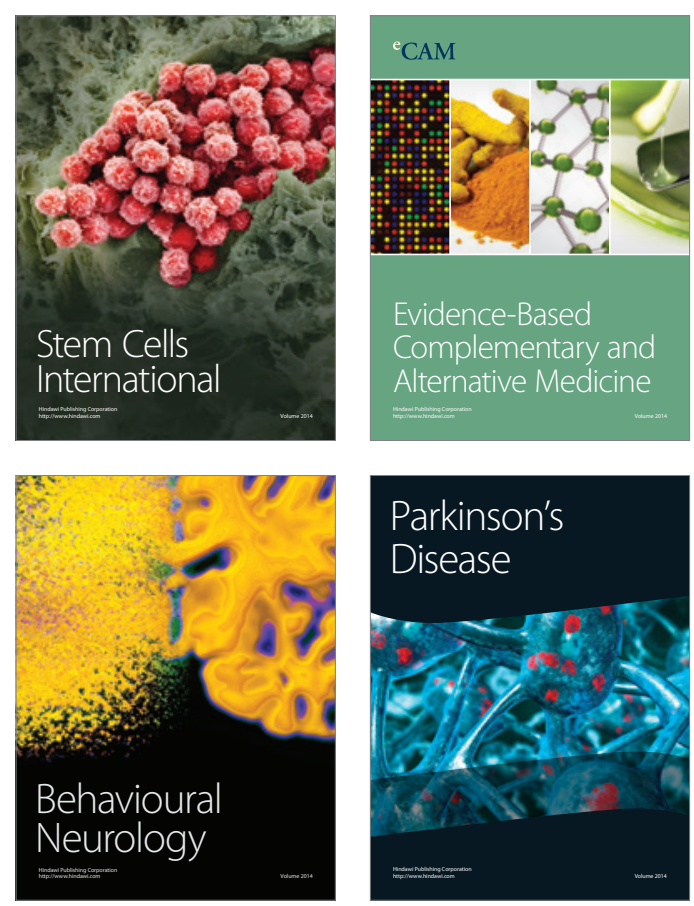

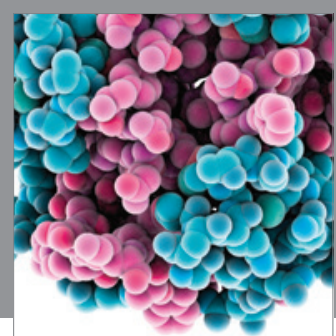

Journal of
Diabetes Research

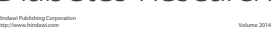

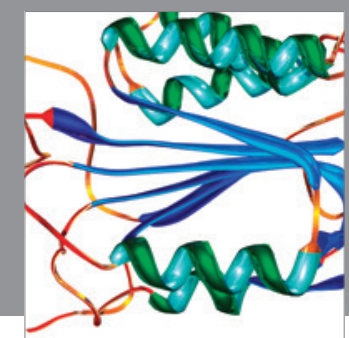

Disease Markers
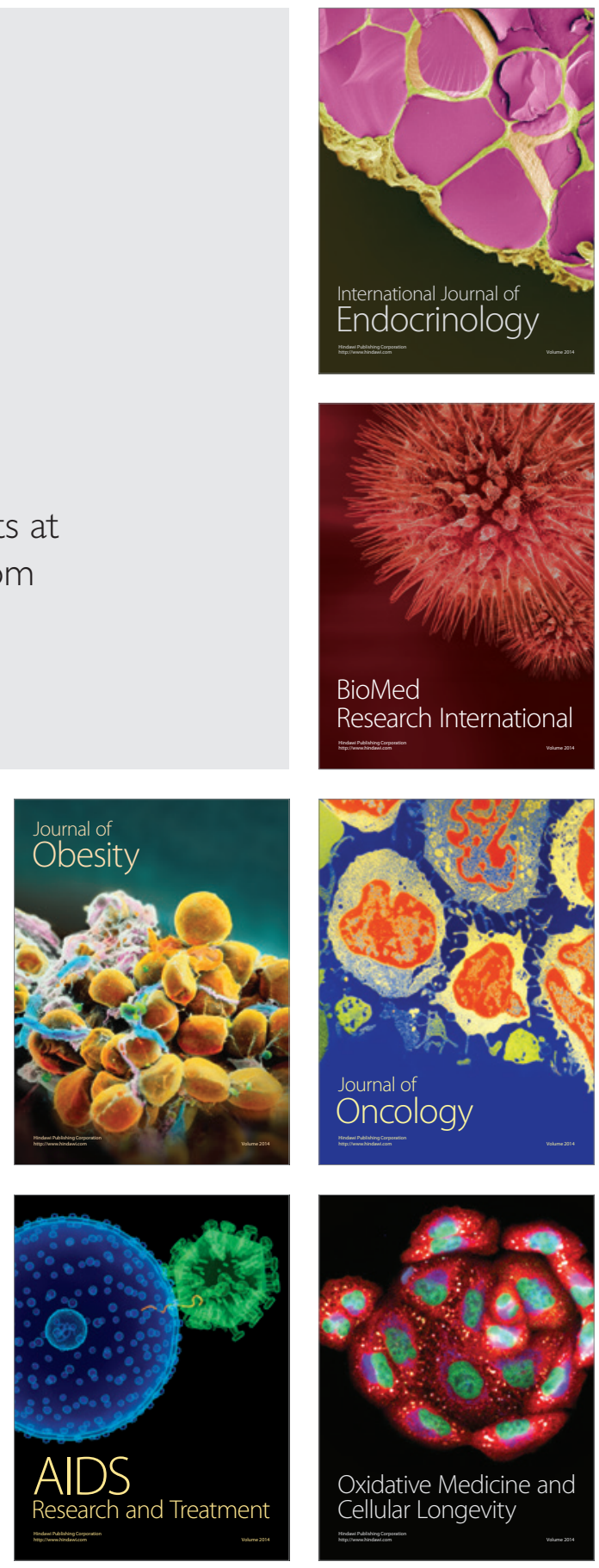